Honam Mathematical J. 36 (2014), No. 1, pp. 001-009

http://dx.doi.org/10.5831/HMJ.2014.36.1.1

\title{
COMMUTATIVE DIAGRAMS WITH FIBER PRODUCTS OF THREE MODULES
}

\author{
SAng-Cho Chung
}

\begin{abstract}
In this paper, we investigate some properties in commutative diagrams with fiber products of three modules.
\end{abstract}

\section{Introduction}

We assume that every ring $R$ is a commutative ring with identity and every module is unitary.

In algebraic K-theory, we can find the concept of fiber products of two $R$-modules([1], [5]). The notion of a fiber product is one that comes up very often in mathematics and logic. We knew various properties of fiber products of two $R$-modules ([1], [3], [4], [5]). In general the definition of fiber products is useful for two modules. In [2], Chung, Kim and Seo gave a generalization of fiber products of modules, that was, we defined the fiber product of three modules and studied some criteria of the fiber product of three $R$-modules(cf. Lemma 2.1, Lemma 2.2, Lemma 2.3).

We introduce the usual definition([1], [5]) of the fiber product of $R$ modules $M_{1}$ and $M_{2}$ over $N$. Let $f_{1}: M_{1} \rightarrow N$ and $f_{2}: M_{2} \rightarrow N$ be homomorphisms of $R$-modules. The fiber product of $M_{1}$ and $M_{2}$ is a triple $\left(M, g_{1}, g_{2}\right)$ where $g_{1}: M \rightarrow N$ and $g_{2}: M \rightarrow M_{2}$ are $R$-linear maps such that $f_{1} g_{1}=f_{2} g_{2}$, and the triple is universal in the sense that given any other triple $\left(M^{\prime}, g_{1}^{\prime}, g_{2}^{\prime}\right)$ of this kind with $f_{1} g_{1}^{\prime}=f_{2} g_{2}^{\prime}$. There is a unique homomorphism $h: M^{\prime} \rightarrow M$ such that $g_{1} h=g_{1}^{\prime}$ and $g_{2} h=g_{2}^{\prime}$.

The purpose of this paper is to investigate some properties in commutative diagrams with fiber products of three $R$-modules in Theorem 2.4 .

Received September 25, 2013. Accepted October 8, 2013.

2010 Mathematics Subject Classification. 13C99.

Key words and phrases. fiber product, commutative diagram. 


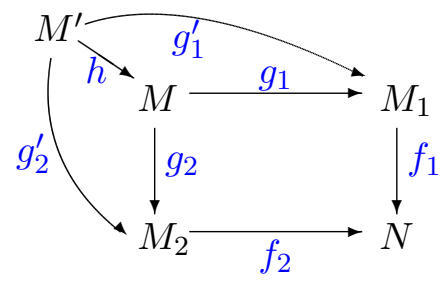

\section{Commutative diagrams with fiber Products}

Firstly, we define the fiber product of three modules. For $R$-modules $M, M_{1}, M_{2}, M_{3}$ and $N$, let $f_{1}: M_{1} \rightarrow N, f_{2}: M_{2} \rightarrow N$ and $f_{3}: M_{3} \rightarrow N$ be $R$-homomorphisms. The fiber product of $M_{1}, M_{2}$ and $M_{3}$ over $N$ is a quadruple $\left(M, g_{1}, g_{2}, g_{3}\right)$ where $g_{1}: M \rightarrow M_{1}, g_{2}: M \rightarrow M_{2}$ and $g_{3}: M \rightarrow M_{3}$ are $R$-homomorphisms such that $f_{1} g_{1}=f_{2} g_{2}=f_{3} g_{3}$, and the quadruple is universal in the sense that given any other quadruple $\left(M^{\prime}, g_{1}^{\prime}, g_{2}^{\prime}, g_{3}^{\prime}\right)$ of this kind with $f_{1} g_{1}^{\prime}=f_{2} g_{2}^{\prime}=f_{3} g_{3}^{\prime}$, there is a unique homomorphism $h: M^{\prime} \rightarrow M$ such that $g_{1} h=g_{1}^{\prime}, g_{2} h=g_{2}^{\prime}$ and $g_{3} h=g_{3}^{\prime}$.

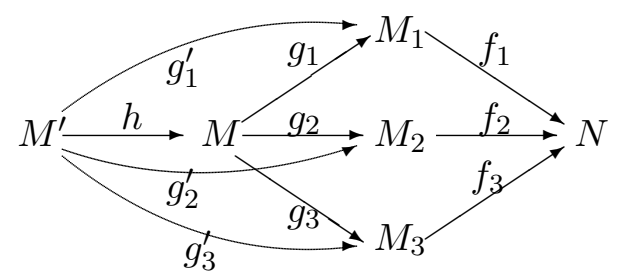

Let $f_{i}: M_{i} \rightarrow N$ for $i=1,2,3$ be $R$-homomorphisms and let $p_{i}$ : $M_{1} \oplus M_{2} \oplus M_{3} \rightarrow M_{i}$ for $i=1,2,3$ be natural projections. Consider the map

$$
\left(f_{1} p_{1}-f_{3} p_{3}, f_{2} p_{2}-f_{3} p_{3}\right): M_{1} \oplus M_{2} \oplus M_{3} \rightarrow N \oplus N .
$$

Then we have

$$
\begin{aligned}
& \operatorname{Im}\left(f_{1} p_{1}-f_{3} p_{3}, f_{2} p_{2}-f_{3} p_{3}\right) \\
&=\left\{\left(f_{1}\left(m_{1}\right)-f_{3}\left(m_{3}\right), f_{2}\left(m_{2}\right)-f_{3}\left(m_{3}\right)\right) \in N \oplus N:\right. \\
&\left.\left(m_{1}, m_{2}, m_{3}\right) \in M_{1} \oplus M_{2} \oplus M_{3}\right\}
\end{aligned}
$$

and

$$
\begin{aligned}
& \operatorname{Ker}\left(f_{1} p_{1}-f_{3} p_{3}, f_{2} p_{2}-f_{3} p_{3}\right) \\
= & \left\{\left(m_{1}, m_{2}, m_{3}\right) \in M_{1} \oplus M_{2} \oplus M_{3}: f_{1}\left(m_{1}\right)=f_{2}\left(m_{2}\right)=f_{3}\left(m_{3}\right)\right\}
\end{aligned}
$$

To obtain our main Theorem, we need the following three Lemmas in $[2]$. 
Lemma 2.1. ([2] Lemma 3.1) Let $R$ be a commutative ring with identity, and let $M, M_{1}, M_{2}, M_{3}$, and $N$ be $R$-modules. Let $f_{i}: M_{i} \rightarrow N$ for $i=1,2,3$ be $R$-homomorphisms and let $p_{i}: M_{1} \oplus M_{2} \oplus M_{3} \rightarrow M_{i}$ for $i=1,2,3$ be natural projections. Let

$M_{123}=\left\{\left(m_{1}, m_{2}, m_{3}\right) \in M_{1} \oplus M_{2} \oplus M_{3}: f_{1}\left(m_{1}\right)=f_{2}\left(m_{2}\right)=f_{3}\left(m_{3}\right)\right\}$.

Then we have the following.

(1) $\left(M_{123},\left.p_{1}\right|_{M_{123}},\left.p_{2}\right|_{M_{123}},\left.p_{3}\right|_{M_{123}}\right)$ is a fiber product of $M_{1}, M_{2}$ and $M_{3}$ over $N$.

(2) Consider the following commutative diagram.

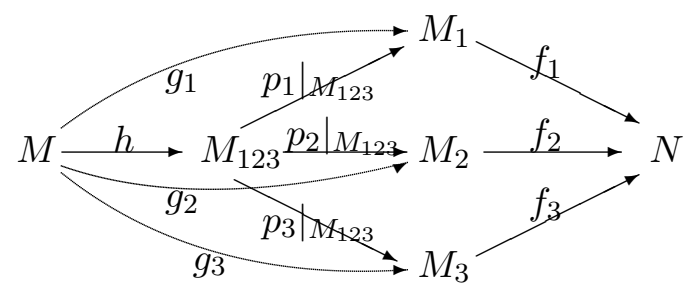

If $\left(M, g_{1}, g_{2}, g_{3}\right)$ is a fiber product of $M_{1}, M_{2}$ and $M_{3}$ over $N$, then we have

(i) there is a unique isomorphism $h: M \rightarrow M_{123}$ such that $\left(\left.p_{1}\right|_{M_{123}}\right) h=g_{1},\left(\left.p_{2}\right|_{M_{123}}\right) h=g_{2}$ and $\left(\left.p_{3}\right|_{M_{123}}\right) h=g_{3}$.

(ii) $\operatorname{Ker}\left(g_{1}\right) \cap \operatorname{Ker}\left(g_{2}\right) \cap \operatorname{Ker}\left(g_{3}\right)=\{0\}$.

Lemma 2.2. ([2] Lemma 3.3) Let $R$ be a commutative ring and

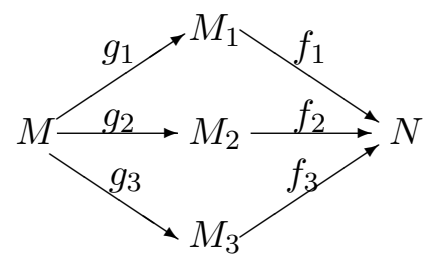

be a commutative diagram of $R$-modules. Then this diagram is a fiber product diagram if and only if for each pair of elements $m_{1} \in M_{1}, m_{2} \in$ $M_{2}, m_{3} \in M_{3}$ with $f_{1}\left(m_{1}\right)=f_{2}\left(m_{2}\right)=f_{3}\left(m_{3}\right)$ there is a unique element $m \in M$ with $g_{1}(m)=m_{1}, g_{2}(m)=m_{2}$ and $g_{3}(m)=m_{3}$.

Lemma 2.3. ([2] Theorem 3.4) Let $R$ be a commutative ring with identity and $M, M_{1}, M_{2}, M_{3}, N$ be $R$-modules. Let $g_{i}: M \rightarrow M_{i}$ for $i=1,2,3$ and $f_{i}: M_{i} \rightarrow N$ for $i=1,2,3$ be $R$-homomorphisms. Let

$$
p_{i}: M_{1} \oplus M_{2} \oplus M_{3} \rightarrow M_{i} \text { for } i=1,2,3
$$

be natural projections. Consider the map

$$
\left(g_{1}, g_{2}, g_{3}\right): M \rightarrow M_{1} \oplus M_{2} \oplus M_{3}
$$


and

$$
\left(f_{1} p_{1}-f_{2} p_{2}, f_{1} p_{1}-f_{3} p_{3}\right): M_{1} \oplus M_{2} \oplus M_{3} \rightarrow N \oplus N .
$$

Then the following are equivalent.

(1) The diagram

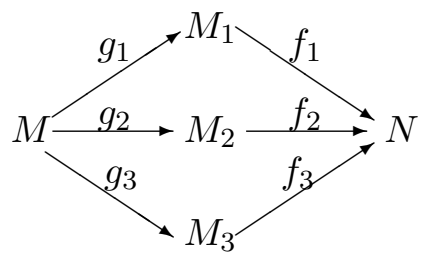

is a fiber product diagram of $M_{1}, M_{2}$ and $M_{3}$ over $N$.

(2) The following sequence

$$
0 \longrightarrow M \stackrel{g}{\longrightarrow} M_{1} \oplus M_{2} \oplus M_{3} \stackrel{f}{\longrightarrow} \operatorname{Im}(f) \longrightarrow 0
$$

is exact, where $g=\left(g_{1}, g_{2}, g_{3}\right)$ and $f=\left(f_{1} p_{1}-f_{2} p_{2}, f_{1} p_{1}-f_{3} p_{3}\right)$.

(3) For the diagram in (1), we have

(i) $\left(g_{1}, g_{2}, g_{3}\right)$ is a monomorphism.

(ii) $\left\{\left(m_{1}, m_{2}, m_{3}\right) \in M_{1} \oplus M_{2} \oplus M_{3}: f_{1}\left(m_{1}\right)=f_{2}\left(m_{2}\right)=f_{3}\left(m_{3}\right)\right\} \subset$ $\operatorname{Im}\left(g_{1}, g_{2}, g_{3}\right)$.

The following Theorem 2.4 is the our main result.

Theorem 2.4. Suppose that each square in the following diagram is a fiber product (or commutative).

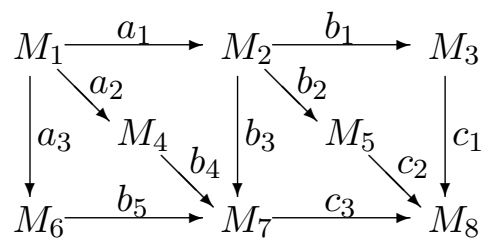

Then we have the following.

(1) The quadruple $\left(M_{1}, a_{1}, a_{2}, a_{3}\right)$ is a fiber product of $M_{2}, M_{4}, M_{6}$ over $M_{8}$ if and only if $c_{3}$ is a monomorphism.

(2) The quadruple $\left(M_{1}, b_{1} a_{1}, a_{2}, a_{3}\right)$ is a fiber product of $M_{3}, M_{4}, M_{6}$ over $M_{8}$, when $a_{2}$ (or $a_{3}$ ), $c_{3}$ are monomorphisms and $b_{1}$ is an epimorphism.

(3) (A commutative case) The quadruple $\left(M_{1}, b_{1} a_{1}, b_{4} a_{2}, a_{3}\right)$ is a fiber product of $M_{3}, M_{7}, M_{6}$ over $M_{8}$, when $c_{1}, c_{3}$ are monomorphisms and $a_{3}$ is an isomorphism. 
(4) The quadruple $\left(M_{1}, b_{1} a_{1}, b_{2} a_{1}, a_{3}\right)$ is a fiber product of $M_{3}, M_{5}, M_{6}$ over $M_{8}$, when $b_{4}$ is an isomorphism.

(5) The quadruple $\left(M_{1}, b_{1} a_{1}, b_{1} a_{2}, b_{3} a_{1}\right)$ is a fiber product of $M_{3}, M_{5}, M_{7}$ over $M_{8}$ if and only if $a_{1}$ is an isomorphism.

Proof: (1) Let

$$
p_{i}: M_{2} \oplus M_{4} \oplus M_{6} \rightarrow M_{i} \text { for } i=2,4,6 .
$$

be natural maps.

$(\Longrightarrow)$ Let $m_{7} \in \operatorname{Ker}\left(c_{3}\right)$. Then we have

$$
c_{3}\left(m_{7}\right)=0=c_{1}(0)=c_{2}(0) .
$$

By Lemma 2.2, there is an element $m_{2} \in M_{2}$ such that

$$
b_{1}\left(m_{2}\right)=0, b_{2}\left(m_{2}\right)=0 \text { and } b_{3}\left(m_{2}\right)=m_{7} .
$$

Then we have

$$
c_{3}\left(b_{3}\left(m_{2}\right)\right)=c_{3}\left(m_{7}\right)=0=c_{3}\left(b_{4}(0)\right)=c_{3}\left(b_{5}(0)\right) .
$$

By the hypothesis and Lemma 2.2, there is an element $m \in M$ such that

$$
a_{1}(m)=m_{2}, a_{2}(m)=0 \text { and } a_{3}(m)=0 .
$$

Then we have

$$
m_{7}=b_{3}\left(m_{2}\right)=b_{3}\left(a_{1}(m)\right)=b_{4}\left(a_{2}(m)\right)=b_{4}(0)=0 .
$$

Therefore $c_{3}$ is a monomorphism.

$(\Longleftarrow)$ Let the map

$$
\left(a_{1}, a_{2}, a_{3}\right): M_{1} \rightarrow M_{2} \oplus M_{4} \oplus M_{6}
$$

where $\left(a_{1}, a_{2}, a_{3}\right)\left(m_{1}\right)=\left(a_{1}\left(m_{1}\right), a_{2}\left(m_{1}\right), a_{3}\left(m_{1}\right)\right)$ for all $m_{1} \in M_{1}$.

Consider a map

$$
\left(c_{3} b_{3} p_{2}-c_{3} b_{4} p_{4}, c_{3} b_{3} p_{2}-c_{3} b_{5} p_{6}\right): M_{2} \oplus M_{4} \oplus M_{6} \rightarrow M_{8} \oplus M_{8} \text {. }
$$

Since $\left(M_{1}, a_{1}, a_{2}, a_{3}\right)$ is a fiber product of $M_{2}, M_{4}, M_{6}$ over $M_{7}$, by Lemma 2.3 the map $\left(a_{1}, a_{2}, a_{3}\right)$ is a monomorphism.

(Method I) Hence it suffices to show that by Lemma 2.3

$$
\operatorname{Ker}\left(c_{3} b_{3} p_{2}-c_{3} b_{4} p_{4}, c_{3} b_{3} p_{2}-c_{3} b_{5} p_{6}\right) \subset \operatorname{Im}\left(a_{1}, a_{2}, a_{3}\right) .
$$

Let $\left(m_{2}, m_{4}, m_{6}\right) \in \operatorname{Ker}\left(c_{3} b_{3} p_{2}-c_{3} b_{4} p_{4}, c_{3} b_{3} p_{2}-c_{3} b_{5} p_{6}\right)$. Then we have

$$
c_{3}\left(b_{3}\left(m_{2}\right)\right)=c_{3}\left(b_{4}\left(m_{4}\right)\right)=c_{3}\left(b_{5}\left(m_{6}\right)\right) .
$$

Since $c_{3}$ is a monomorphism, we have

$$
b_{3}\left(m_{2}\right)=b_{4}\left(m_{4}\right)=b_{5}\left(m_{6}\right) \text {. }
$$


By Lemma 2.2, there is an element $m_{1} \in M_{1}$ such that

$$
a_{1}\left(m_{1}\right)=m_{2}, a_{2}\left(m_{1}\right)=m_{4} \text { and } a_{3}\left(m_{1}\right)=m_{6} .
$$

Therefore

and hence

$$
\left(m_{2}, m_{4}, m_{6}\right)=\left(a_{1}\left(m_{1}\right), a_{2}\left(m_{1}\right), a_{3}\left(m_{1}\right)\right)
$$

$$
\operatorname{Ker}\left(c_{3} b_{3} p_{2}-c_{3} b_{4} p_{4}, c_{3} b_{3} p_{2}-c_{3} b_{5} p_{6}\right)=\operatorname{Im}\left(a_{1}, a_{2}, a_{3}\right) .
$$

(Method II) Consider a map

$$
\left(b_{3} p_{2}-b_{4} p_{4}, b_{3} p_{2}-b_{5} p_{6}\right): M_{2} \oplus M_{4} \oplus M_{6} \rightarrow M_{7} \oplus M_{7} .
$$

Then since $c_{3}$ is a monomorphism,

$\operatorname{Ker}\left(b_{3} p_{2}-b_{4} p_{4}, b_{3} p_{2}-b_{5} p_{6}\right)=K e r\left(c_{3} b_{3} p_{2}-c_{3} b_{4} p_{4}, c_{3} b_{3} p_{2}-c_{3} b_{5} p_{6}\right)$.

Hence by the hypothesis we have

$$
\begin{aligned}
M_{1} & \cong \operatorname{Im}\left(a_{1}, a_{2}, a_{3}\right) \\
& =\operatorname{Ker}\left(b_{3} p_{2}-b_{4} p_{4}, b_{3} p_{2}-b_{5} p_{6}\right) \\
& =\operatorname{Ker}\left(c_{3} b_{3} p_{2}-c_{3} b_{4} p_{4}, c_{3} b_{3} p_{2}-c_{3} b_{5} p_{6}\right) .
\end{aligned}
$$

The result follows from Lemma 2.3.

(2) For natural maps

$$
p_{i}: M_{3} \oplus M_{4} \oplus M_{6} \rightarrow M_{i} \text { for } i=3,4,6
$$

consider a map

$$
\left(c_{1} p_{3}-c_{3} b_{4} p_{4}, c_{1} p_{3}-c_{3} b_{5} p_{6}\right): M_{3} \oplus M_{4} \oplus M_{6} \rightarrow M_{8} \oplus M_{8} .
$$

Since $a_{2}$ (or $\left.a_{3}\right)$ is a monomorphism, $\left(b_{1} a_{1}, a_{2}, a_{3}\right)$ is a monomorphism. Next we show that

$$
\operatorname{Ker}\left(c_{1} p_{3}-c_{3} b_{4} p_{4}, c_{1} p_{3}-c_{3} b_{5} p_{6}\right) \subset \operatorname{Im}\left(b_{1} a_{1}, a_{2}, a_{3}\right) .
$$

Let $\left(m_{3}, m_{4}, m_{6}\right) \in \operatorname{Ker}\left(c_{1} p_{3}-c_{3} b_{4} p_{4}, c_{1} p_{3}-c_{3} b_{5} p_{6}\right)$. Then we have

$$
c_{1}\left(m_{3}\right)=c_{3}\left(b_{4}\left(m_{4}\right)\right)=c_{3}\left(b_{5}\left(m_{6}\right)\right) .
$$

Since $b_{1}$ is surjective, there is an element $m_{2} \in M_{2}$ such that

$$
b_{1}\left(m_{2}\right)=m_{3} .
$$

Then we have

$$
c_{3}\left(b_{3}\left(m_{2}\right)\right)=c_{1}\left(b_{1}\left(m_{2}\right)\right)=c_{1}\left(m_{3}\right)=c_{3}\left(b_{4}\left(m_{4}\right)\right)=c_{3}\left(b_{5}\left(m_{6}\right)\right) .
$$

Since $c_{3}$ is a monomorphism, we have

$$
b_{3}\left(m_{2}\right)=b_{4}\left(m_{4}\right)=b_{5}\left(m_{6}\right) .
$$


By Lemma 2.2, there is an element $m_{1} \in M_{1}$ such that

$$
a_{1}\left(m_{1}\right)=m_{2}, a_{2}\left(m_{1}\right)=m_{4} \text { and } a_{3}\left(m_{1}\right)=m_{6} .
$$

Then we have $b_{1}\left(a_{1}\left(m_{1}\right)\right)=b_{1}\left(m_{2}\right)=m_{3}$. Hence

$$
\left(m_{3}, m_{4}, m_{6}\right)=\left(b_{1}\left(a_{1}\left(m_{1}\right)\right), a_{2}\left(m_{1}\right), a_{3}\left(m_{1}\right)\right) .
$$

Thus we have

$$
\operatorname{Im}\left(b_{1} a_{1}, a_{2}, a_{3}\right)=\operatorname{Ker}\left(c_{1} p_{3}-c_{3} b_{4} p_{4}, c_{1} p_{3}-c_{3} b_{5} p_{6}\right) .
$$

(3) For natural maps

$$
p_{i}: M_{3} \oplus M_{7} \oplus M_{6} \rightarrow M_{i} \text { for } i=3,7,6
$$

consider a map

$$
\left(c_{1} p_{3}-c_{3} p_{7}, c_{1} p_{3}-c_{3} b_{5} p_{6}\right): M_{3} \oplus M_{7} \oplus M_{6} \rightarrow M_{8} \oplus M_{8} .
$$

Since $a_{3}$ is a monomorphism, $\left(b_{1} a_{1}, b_{4} a_{2}, a_{3}\right)$ is a monomorphism. Next we show that

$$
\operatorname{Ker}\left(c_{1} p_{3}-c_{3} p_{7}, c_{1} p_{3}-c_{3} b_{5} p_{6}\right) \subset \operatorname{Im}\left(b_{1} a_{1}, b_{4} a_{2}, a_{3}\right) .
$$

Let $\left(m_{3}, m_{7}, m_{6}\right) \in \operatorname{Ker}\left(c_{1} p_{3}-c_{3} p_{7}, c_{1} p_{3}-c_{3} b_{5} p_{6}\right)$. Then we have

$$
c_{1}\left(m_{3}\right)=c_{3}\left(m_{7}\right)=c_{3}\left(b_{5}\left(m_{6}\right)\right) .
$$

Since $c_{3}$ is a monomorphism, we have $m_{7}=b_{5}\left(m_{6}\right)$. Since $a_{3}$ is an epimorphism, there is an element $m_{1} \in M_{1}$ such that

$$
a_{3}\left(m_{1}\right)=m_{6} \text {. }
$$

Then we have

$$
m_{7}=b_{5}\left(m_{6}\right)=b_{5}\left(a_{3}\left(m_{1}\right)\right)=b_{4}\left(a_{2}\left(m_{1}\right)\right)=b_{3}\left(a_{1}\left(m_{1}\right)\right) .
$$

Hence we have

$$
c_{1}\left(m_{3}\right)=c_{3}\left(m_{7}\right)=c_{3}\left(b_{3}\left(a_{1}\left(m_{1}\right)\right)\right)=c_{1}\left(b_{1}\left(a_{1}\left(m_{1}\right)\right)\right) .
$$

Since $c_{1}$ is a monomorphism, we have

$$
m_{3}=b_{1}\left(a_{1}\left(m_{1}\right)\right) \text {. }
$$

Hence we have

$$
\left(m_{3}, m_{7}, m_{6}\right)=\left(b_{1}\left(a_{1}\left(m_{1}\right)\right), b_{4}\left(a_{2}\left(m_{1}\right)\right), a_{3}\left(m_{1}\right)\right) .
$$

Thus we have

$$
\operatorname{Im}\left(b_{1} a_{1}, b_{4} a_{2}, a_{3}\right)=\operatorname{Ker}\left(c_{1} p_{3}-c_{3} p_{7}, c_{1} p_{3}-c_{3} b_{5} p_{6}\right) .
$$

(4) For natural maps

$$
p_{i}: M_{3} \oplus M_{5} \oplus M_{6} \rightarrow M_{i} \text { for } i=3,5,6
$$


consider a map

$$
\left(c_{1} p_{3}-c_{2} p_{5}, c_{1} p_{3}-c_{3} b_{5} p_{6}\right): M_{3} \oplus M_{5} \oplus M_{6} \rightarrow M_{8} \oplus M_{8} .
$$

Let $m_{1} \in \operatorname{Ker}\left(b_{1} a_{1}, b_{2} a_{1}, a_{3}\right)$. Then we have

$$
b_{1}\left(a_{1}\left(m_{1}\right)\right)=0, b_{2}\left(a_{1}\left(m_{1}\right)\right)=0, a_{3}\left(m_{1}\right)=0 .
$$

Thus

$$
0=b_{5}\left(a_{3}\left(m_{1}\right)\right)=b_{4}\left(a_{2}\left(m_{1}\right)\right)=b_{3}\left(a_{1}\left(m_{1}\right)\right) .
$$

Therefore we have

$$
a_{1}\left(m_{1}\right) \in \operatorname{Ker}\left(b_{1}\right) \cap \operatorname{Ker}\left(b_{2}\right) \cap \operatorname{Ker}\left(b_{3}\right) .
$$

By Lemma 2.1

$$
a_{1}\left(m_{1}\right)=0 .
$$

Since $b_{4}$ is a monomorphism, we have $a_{2}\left(m_{1}\right)=0$ and then

$$
m_{1} \in \operatorname{Ker}\left(a_{1}\right) \cap \operatorname{Ker}\left(a_{2}\right) \cap \operatorname{Ker}\left(a_{3}\right) .
$$

By Lemma 2.1

$$
m_{1}=0 \text {. }
$$

Hence $\left(b_{1} a_{1}, b_{2} a_{1}, a_{3}\right)$ is a monomorphism.

Next we show that

$$
\operatorname{Ker}\left(c_{1} p_{3}-c_{2} p_{5}, c_{1} p_{3}-c_{3} b_{5} p_{6}\right) \subset \operatorname{Im}\left(b_{1} a_{1}, b_{2} a_{1}, a_{3}\right) .
$$

Since $\left(c_{1} p_{3}-c_{2} p_{5}, c_{1} p_{3}-c_{3} b_{5} p_{6}\right)\left(b_{1} a_{1}, b_{2} a_{1}, a_{3}\right)=(0,0)$, we have

$$
\operatorname{Im}\left(b_{1} a_{1}, b_{2} a_{1}, a_{3}\right) \subset \operatorname{Ker}\left(c_{1} p_{3}-c_{2} p_{5}, c_{1} p_{3}-c_{3} b_{5} p_{6}\right) .
$$

Let $\left(m_{3}, m_{5}, m_{6}\right) \in \operatorname{Ker}\left(c_{1} p_{3}-c_{2} p_{5}, c_{1} p_{3}-c_{3} b_{5} p_{6}\right)$. Then we have

$$
c_{1}\left(m_{3}\right)=c_{2}\left(m_{5}\right)=c_{3}\left(b_{5}\left(m_{6}\right)\right) .
$$

By Lemma 2.2, there is an element $m_{2} \in M_{2}$ such that

$$
b_{1}\left(m_{2}\right)=m_{3}, b_{2}\left(m_{2}\right)=m_{5} \text { and } b_{3}\left(m_{2}\right)=b_{5}\left(m_{6}\right) \text {. }
$$

Since $b_{4}$ is an epimorphism, there is an element $m_{4} \in M_{4}$ such that

$$
b_{4}\left(m_{4}\right)=b_{3}\left(m_{2}\right) .
$$

Then we have

$$
b_{3}\left(m_{2}\right)=b_{4}\left(m_{4}\right)=b_{5}\left(m_{6}\right) .
$$

By Lemma 2.2, there is an element $m_{1} \in M_{1}$ such that

$$
a_{1}\left(m_{1}\right)=m_{2}, a_{2}\left(m_{1}\right)=m_{4} \text { and } a_{3}\left(m_{1}\right)=m_{6} .
$$

Then we have

$b_{1}\left(a_{1}\left(m_{1}\right)\right)=b_{1}\left(m_{2}\right)=m_{3}, b_{2}\left(a_{1}\left(m_{1}\right)\right)=b_{2}\left(m_{2}\right)=m_{5}$ and $a_{1}\left(m_{1}\right)=m_{6}$. 
Hence

$$
\left(m_{3}, m_{5}, m_{6}\right)=\left(b_{1}\left(a_{1}\left(m_{1}\right)\right), b_{2}\left(a_{1}\left(m_{1}\right)\right), a_{3}\left(m_{1}\right)\right) .
$$

Thus we have

$$
\operatorname{Im}\left(b_{1} a_{1}, b_{2} a_{1}, a_{3}\right)=\operatorname{Ker}\left(c_{1} p_{3}-c_{2} p_{5}, c_{1} p_{3}-c_{3} b_{5} p_{6}\right) .
$$

(5) It follows from Lemma 2.1.

\section{References}

[1] H. Bass, Algebraic K-Theory, W. A. Benjamin, Inc. 1968.

[2] S. C. Chung, S. A. Kim, and G. S. Seo, Some properties of fiber products of three modules, Honam Mathematical J. 32(2) (2010), 239-253.

[3] E. Kunz, Introduction to Commutative Algebra and Algebraic Geometry, Birkhäuser Boston, 1985.

[4] S. Mandal, Projective Modules and Complete Intersections, Lecture Notes in Mathematics 1672, Springer-Verlag Berlin Heidelberg, 1997.

[5] J. Milnor, Introduction to Algebraic K-Theory, Princeton University Press, 1971.

\section{Sang-Cho Chung}

Department of Mathematics Education, Mokwon University,

Taejon 302-729, Korea.

E-mail : math888@naver.com 